Original article

\title{
Effects of storage time and restoration depth on instrumental color adjustment potential of universal resin composites
}

\author{
Sinem Akgül1), Ceyda Gündoğdu ${ }^{2)}$, and Oya Bala ${ }^{1)}$ \\ Department of Restorative Dentistry, Faculty of Dentistry, Gazi University, Ankara, Turkey \\ ${ }^{2}$ Department of Restorative Dentistry, Faculty of Dentistry, Istanbul Medipol University, Istanbul, Turkey
}

(Received June 21, 2021; Accepted October 29, 2021)

\begin{abstract}
Purpose: To determine the instrumental color adjustment potential (CAPI) of 3 universal composites at 2 time intervals and 2 depths.

Methods: Omnichroma, Estelite Sigma Quick, and Filtek Universal Restorative were tested. Two types of specimens were prepared. For dual specimens, Class V restorations were created in anterior denture teeth and restored with resin composites. For single specimens, replications were fabricated with resin composites $(n=10)$. Unrestored teeth were used for comparison. Color was measured with a spectrophotometer at $24 \mathrm{~h}$ and 1 month, and CAP-I was calculated. Data were compared by analysis of variance followed by the Student $t$-test and paired sample $t$-test.

Results: The CAP-I of tested materials ranged from 0.11 to 0.27 at $24 \mathrm{~h}$ and from 0.60 to 0.76 at 1 month. At $24 \mathrm{~h}$, Omnichroma yielded the highest values, followed by Estelite Sigma Quick and Filtek Universal Restorative $(P<0.05)$. CAP-I was significantly higher at 1 month than at $24 \mathrm{~h}$ in all groups. In all groups, restorations with a depth of $3.0 \mathrm{~mm}$ yielded better results than restorations with a depth of $2.0 \mathrm{~mm}(P<0.05)$.

Conclusion: Storage time and restoration depth were positively associated with CAP-I. Single-shade and multi-shade universal composites are good alternatives for color-matched, esthetically satisfying restorations.
\end{abstract}

Keywords: blending effect, color adjustment potential, spectrophotometer, universal resin composite

\section{Introduction}

Resin composites are commonly used in modern dentistry, as they provide natural esthetics with a conservative approach for managing tooth structure loss and modification of tooth color and shape [1]. Esthetic considerations are mostly related to precise color matching and sufficient adaptation between the restoration and adjacent dental hard tissues. In addition, restoration location and contour and tooth texture and color are other important factors for successful outcomes [2].

Since the first use of resin composites in dentistry, their esthetics and mechanical properties have improved considerably. The color-related properties of materials - color compatibility, color stability, and color interactions - are key factors in evaluating the clinical performance of resin composites [3]. Some available resin composites are marketed according to Vita Classic shade guide and are available in multiple enamel, dentin, body, and opaque shades that mimic the optical properties of dental tissues with varying translucency, opacity, filler content, shape, and size [4]. Although only four shades were available initially, now 32 shades are offered [5].

The layering technique used for esthetic restorations was introduced in 1980 [6]. This technique allows restorations to be performed with resin composites of different shades - to simulate the optical properties of dental tissues - although it requires more technical sensitivity, processing steps,

Correspondence to Dr. Sinem Akgül, Department of Restorative Dentistry, Faculty of Dentistry, Gazi University, Emek, Ankara TR-06910, Turkey

Fax: +90-312-223-92-26 E-mail: sinemakipek@gmail.com

Color figures can be viewed in the online issue at J-STAGE.

doi.org/10.2334/josnusd.21-0290

DN/JST.JSTAGE/josnusd/21-0290 and chairside time. Color contrast and color assimilation are terms used to quantify color change against adjacent structures in opposite directions. Color contrast expresses how a uniform color region in a different color environment looks different from the surrounding color, while color assimilation expresses how an applied color harmonizes with adjacent structures [7]. Color assimilation is also referred to as blending effect (BE) (the term "chameleon effect" is used in dental parlance to describe this phenomenon), which refers to the ability of a dental material to acquire a color similar to that of the adjacent dental tissues under isolation [8].

The methods used for color assessment in the literature are mainly classified as visual assessment and instrumental assessment. Visual evaluations are commonly used in clinical conditions because of their easy application by clinicians. However, this method can be affected by factors such as the patient's cosmetics and clothes, the type of external light source, the clinician's age and experience, and even normal vision differences among clinicians of the same age and experience level [9]. Therefore, objective color analysis with instrumental methods and spectrophotometers is sometimes used. Different methods have been used for instrumental evaluation of the color matching and color stability potential of restorative materials $[2,10]$. The CIELAB color space is a widely used method that was introduced by the Commission Internationale de I'Eclairage (CIE, International Commission on Illumination) and yields values with $\mathrm{L}^{*}$, $\mathrm{a}^{*}$, and $\mathrm{b}^{*}$ coordinates [11].

Under clinical conditions, color selection and the use of colors similar to those of dental tissues are important, difficult, and operator-dependent issues. In layering technique, the operator must apply materials of different shades to mimic tooth tissues. Therefore, minimizing shade selection, simplifying protocols, and reducing chair-side time are desirable when choosing a restorative material in dental practice [12]. The available materials and so-called universal composites have a single shade that mimics the esthetic properties of dental tissues [3]. Although these resin composites have a single shade, they are purported to match with different tooth colors. The manufacturers maintain that these resin composites also have improved color adjustment potential (CAP), a term that describes and quantifies the interaction between the perceptual and physical components of blending obtained visually and by color measurements with devices, respectively [2]. A threshold for effective blending has been identified, and higher values indicate better CAP [3].

Recently launched universal restorative materials focus on color matching between materials and dental tissues to achieve adequate esthetics, especially in anterior restorations. Few studies have investigated the blending capacity of universal resin composites [12], and no published study evaluated the color adjustment potential of single-shade and multi-shade resin composites in relation to storage time and restoration depth. This study assessed the instrumental color adjustment potential (CAP-I) of 3 universal resin composites in Class $\mathrm{V}$ restorations on acrylic denture teeth at $24 \mathrm{~h}$ and 1 month. The null hypothesis was that storage time and restoration depth would have no effect on the CAP-I of the universal restorative materials.

\section{Materials and Methods}

\section{Specimen preparation}

Three commercially available universal resin composites were evaluated (Table 1). Three types of specimens were prepared (Fig. 1): dual specimens (denture tooth restored with tested resin composite), single specimens 
Table 1 Universal resin composites tested in this study

\begin{tabular}{|c|c|c|c|c|c|c|c|}
\hline \multirow[t]{2}{*}{ Product name, Manufacturer } & \multirow[t]{2}{*}{ Lot number } & \multirow[t]{2}{*}{ Shade } & \multicolumn{2}{|c|}{ Filler content } & \multirow[t]{2}{*}{ Filler size } & \multirow[t]{2}{*}{ Filler type } & \multirow[t]{2}{*}{ Monomer } \\
\hline & & & $\mathrm{wt} \%$ & vol\% & & & \\
\hline $\begin{array}{l}\text { Omnichroma, Tokuyama Dental } \\
\text { Corp., Tokyo, Japan }\end{array}$ & $06 \mathrm{E} 39$ & - & 79.0 & 68.0 & $260 \mathrm{~nm}$ & spheric silica zirconia filler & UDMA, TEGDMA \\
\hline $\begin{array}{l}\text { Estelite Sigma Quick, Tokuyama } \\
\text { Dental Corp. }\end{array}$ & 170EY9 & A3 & 82.0 & 71.0 & $100-300 \mathrm{~nm}$ & silica zirconia filler & Bis-GMA, TEGDMA \\
\hline $\begin{array}{l}\text { Filtek Universal Restorative, } 3 \mathrm{M} \\
\text { ESPE, Saint Paul, MN, USA }\end{array}$ & NA66211 & $\mathrm{A} 3$ & 76.5 & 58.4 & $\begin{array}{l}\text { composed of } 20-\mathrm{nm} \text { silica, } \\
\text { 4- to } 11 \text {-nm zirconia, and } \\
\text { 100-nm ytterbium trifluoride }\end{array}$ & $\begin{array}{l}\text { silica filler, zirconia filler, } \\
\text { ytterbium trifluoride }\end{array}$ & $\begin{array}{l}\text { AUDMA, AFM, diurethane- } \\
\text { DMA, } 1,12 \text { dodecane DMA }\end{array}$ \\
\hline
\end{tabular}

UDMA, urethane dimethacrylate; TEGDMA, triethylene glycol dimethacrylate; Bis-GMA, bisphenol A diglycidylmethacrylate; AUDMA, aromatic urethane dimethacrylate; AFM, addition-fragmentation monomer

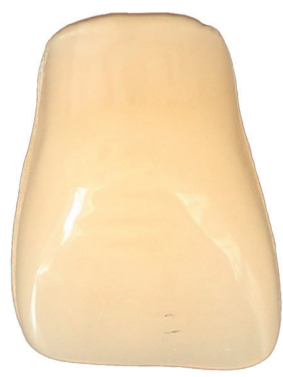

a

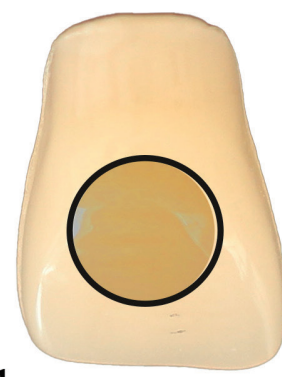

b

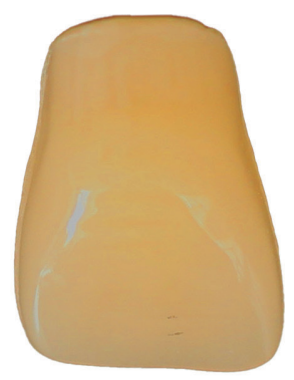

$\mathrm{C}$

Fig. 1 Schematic representation of specimen types: a) Unrestored denture tooth (control specimen), b) Denture tooth restored with resin composites (dual specimen), c) Denture tooth replicated with resin composite (single specimen)

(denture tooth replicated with tested resin composite), and control specimens (unrestored denture tooth).

Seventy maxillary central, acrylic denture teeth (ErayDent, Ankara, Turkey) with a spectrophotometer-confirmed baseline color of A3 (Vita Easyshade V, Vita Zahnfabrik, Bad Sackingen, Germany) (10 mm height, $8 \mathrm{~mm}$ width) were selected. Initial spectral measurements were performed from the middle third of the labial side of the denture tooth, in accordance with the CIELAB system and Vita scale. Ten denture teeth were selected for the control groups, not restored, and directly stored at $37^{\circ} \mathrm{C}$ and $100 \%$ relative humidity. The remaining 60 teeth were designated as dual specimens and were randomly divided into 2 groups according to the restoration depth, i.e., $2.0 \mathrm{~mm}$ and $3.0 \mathrm{~mm}(n=30)$. In both groups, after cavity preparations with a diamond fissure bur without bevel, the color parameters ( $\mathrm{L}, \mathrm{a}$, and $\mathrm{b}$ values) of the cavity floors were measured with a spectrophotometer. During the experiment, all color measurements were performed at the same temperature and with the same background and light exposure, to standardize the color evaluations.

In the 2.0-mm groups, standardized, circular $6.0-\mathrm{mm}$ diameter cavities were prepared in the center of each denture tooth by a single operator, and cavity floor color measurements were performed. Before the restorations, cavities were treated with an adhesive (Tokuyama Universal Bond, Tokuyama Dental Corp., Tokyo, Japan), in accordance with manufacturers' instructions. After that, teeth were divided into 3 subgroups according to the resin composites used $(n=10)$. The groups were Omnichroma (Tokuyama Dental Corp.), Estelite Sigma Quick (Tokuyama Dental Corp.), and Filtek Universal Restorative (3M ESPE, Saint Paul, MN, USA). In each group, cavities were restored according to the manufacturers' instructions.

In the $3.0-\mathrm{mm}$ groups, after cavity preparations and color measurements were performed, teeth were randomly divided into 3 subgroups, as mentioned above, and restored incrementally in 2.0-mm layers with resin composites. In all groups, a polyester strip (Mylar, DuPont, Wilmington, DE, USA) was applied on top of the restorations, to eliminate the oxygen inhibition layer. After that, polymerization was performed with a lightemitting diode (LED) light-curing unit with a light intensity of 1,000 mW/ $\mathrm{cm}^{2}$ (Elipar Free Light 2, 3M ESPE) in direct contact with the specimen for $10 \mathrm{~s}$, in accordance with the manufacturer's instructions. A radiometer (Bluephase Meter II, Ivoclar Vivadent, Amherst, NY, USA) was used to measure polymerization lamp output after each specimen was prepared during the experiment. Restorations were polished with aluminum oxidecoated polishing disks (Sof-Lex Contouring and Polishing Disks, 3M
ESPE) with medium grit for 10,000 rpm and with fine and super-fine grits for $30,000 \mathrm{rpm}$ for $30 \mathrm{~s}$ by the same operator. Each specimen was rinsed with water after use of each disk until the final polishing step and then air-dried.

For the single specimens, a clear silicon mold was used to replicate the denture tooth with each evaluated resin composite $(n=10)$, for a total of 30 single specimens. Replicated specimens were polished in the manner described above.

Specimens were then stored at $37^{\circ} \mathrm{C}$ and $100 \%$ relative humidity during the experiment.

\section{Instrumental evaluation}

A portable clinical spectrophotometer (Vita Easyshade V, Vita Zahnfabrik) was used for the color measurements. Each tooth was placed on a white silicone base (Rapid; Coltène Whaledent AG, Altstätten, Switzerland), with the labial surface on top before color measurement. Color measurements of the specimens were evaluated at $24 \mathrm{~h}$ and 1 month. Specimens were stored in distilled water under the same daylight and temperature, in accordance with the CIELAB system, where $L^{*}$ represents the luminosity of the material on a scale of 0 (black) to 100 (white), a* indicates the hue and chroma on the red-green axis, and $b *$ represents the hue and chroma on the yellow-blue axis. The spectrophotometer was calibrated in compliance with the manufacturer's instructions before each measurement. The color difference between two specimens or two time periods was represented by $\Delta \mathrm{E}^{*}$ and calculated as follows [11]:

$\Delta \mathrm{E}^{*}=\left[\left(\Delta \mathrm{L}^{*}\right)^{2}+\left(\Delta \mathrm{a}^{*}\right)^{2}+\left(\Delta \mathrm{b}^{*}\right)^{2}\right]^{1 / 2}$

A newly created calculation system makes it possible to measure the instrumental color adjustment potential of the material, CAP-I, which is calculated using the following equation in accordance with the CIELAB system:

CAP-I $=1-\left(\Delta \mathrm{E}_{\text {DUAL }} / \Delta \mathrm{E}_{\text {SINGLE }}\right)$

$\Delta \mathrm{E}^{*}{ }_{\text {SINGLE }}$ and $\Delta \mathrm{E}^{*}{ }_{\text {DUAL }}$ correspond to the color difference among the single specimens (those replicated with the tested resin composite) vs an unrestored denture tooth and among dual specimens (in which a denture tooth is restored with resin composite) vs an unrestored denture tooth, respectively.

\section{Statistical analysis}

IBM SPSS Statistics 22 for Windows (IBM SPSS Inc., Chicago, IL, USA) was used for statistical analysis. The sample size was determined on the 
Table 2 Mean and standard deviations of color differences among dual and single specimens and CAP-I values of the tested materials at $24 \mathrm{~h}$ and $1 \mathrm{month}$

\begin{tabular}{|c|c|c|c|c|c|c|}
\hline & \multicolumn{3}{|c|}{ After $24 \mathrm{~h}$} & \multicolumn{3}{|c|}{ After 1 month } \\
\hline & Dual & Single & CAP-I & Dual & Single & CAP-I \\
\hline \multicolumn{7}{|l|}{$2.0 \mathrm{~mm}$} \\
\hline Omnichroma & $6.03(1.44)$ & $7.45(1.26)$ & $0.20(0.19)^{\mathrm{a}}$ & $11.86(1.99)$ & $32.52(0.28)$ & $0.64(0.70)^{\mathrm{A}}$ \\
\hline Estelite Sigma Quick & $7.55(1.59)$ & $8.74(1.18)$ & $0.15(0.30)^{\mathrm{b}}$ & $6.61(1.14)$ & $27.35(0.71)$ & $0.74(0.78)^{\mathrm{B}}$ \\
\hline Filtek Universal Restorative & $6.25(1.11)$ & $7.03(1.17)$ & $0.11(0.20)^{\mathrm{c}}$ & $5.46(1.31)$ & $15.76(1.04)$ & $0.60(0.69)^{\mathrm{c}}$ \\
\hline \multicolumn{7}{|l|}{$3.0 \mathrm{~mm}$} \\
\hline Omnichroma & $7.48(0.92)$ & $8.50(1.18)$ & $0.27(0.33)^{\mathrm{d}}$ & $9.42(1.30)$ & $32.52(0.28)$ & $0.71(0.75)^{\mathrm{D}}$ \\
\hline Estelite Sigma Quick & $8.73(1.11)$ & $10.99(0.61)$ & $0.21(0.29)^{\mathrm{a}}$ & $7.15(1.17)$ & $27.35(0.71)$ & $0.76(0.80)^{\mathrm{B}}$ \\
\hline Filtek Universal Restorative & $9.68(1.58)$ & $11.54(1.07)$ & $0.16(0.23)^{b}$ & $6.28(1.48)$ & $15.76(1.04)$ & $0.65(0.70)^{A}$ \\
\hline
\end{tabular}

The presence of the same superscript letters in a column indicates no significant differences between tested groups after $24 \mathrm{~h}$ and after 1 month, as determined by analysis of variance followed by the paired sample $t$-test $(P<0.05)$

Table 3 Mean CAP-I values (SD) at different restoration depths of the tested materials

\begin{tabular}{llccc}
\hline \multirow{2}{*}{ Omnichroma } & & $2.0 \mathrm{~mm}$ depth & $3.0 \mathrm{~mm}$ depth & \\
\cline { 3 - 4 } & & CAP-I & CAP-I & \\
\hline \multirow{3}{*}{ Estelite Sigma Quick } & after 24 h & $0.20(0.19)$ & $0.27(0.33)$ & $0.048^{*}$ \\
& after 1 month & $0.64(0.70)$ & $0.71(0.75)$ & $0.026^{*}$ \\
Filtek Universal Restorative & after 24 h & $0.15(0.30)$ & $0.21(0.29)$ & $0.000^{*}$ \\
& after 1 month & $0.74(0.78)$ & $0.76(0.80)$ & $0.048^{*}$ \\
& after 24 h & $0.11(0.20)$ & $0.16(0.23)$ & $0.000^{*}$ \\
& after 1 month & $0.60(0.69)$ & $0.65(0.70)$ & $0.000^{*}$ \\
\hline
\end{tabular}

${ }^{*} P<0.05$ indicates a significant difference between groups in each row, as determined by the Student $t$-test.

basis of an effect size (f) of 1.30 , an $\alpha$ of 0.05 , a $\beta$ of 0.20 , a power of 0.85 , and the number of groups. Power analysis suggested that 10 samples would be adequate for effective measurement of color. The normality of the data distribution was analyzed with the Shapiro-Wilk test $(P>0.05)$; the Levene test indicated homogeneity among variances $(P>0.05)$.

CAP-I values were compared by analysis of variance (ANOVA), and mean differences were compared with the Student $t$-test to evaluate the effect of restoration depth $(P<0.05)$. The paired sample $t$-test was used to evaluate the effect of storage time on CAP-I at the 0.05 level of significance.

\section{Results}

Mean color difference $\left(\Delta \mathrm{E}^{*}\right)$ and standard deviation for dual and single specimens, and the corresponding CAP-I at $24 \mathrm{~h}$ and $1 \mathrm{month}$, are shown in Table 2. When the CAP-I of the tested restorative materials was analyzed with instruments, a CAP of 0.20 was used as the threshold for effective blending [3] because it corresponds to a $20.0 \%$ reduction in the dual column, as compared with the single column, in Table 2 . The color adjustment potential of tested restorative materials was 0.11 to 0.27 at $24 \mathrm{~h}$ and 0.60 to 0.76 at 1 month. After 1 month of storage in distilled water, all materials had acceptable CAP-I values, i.e., greater than 0.20 .

After $24 \mathrm{~h}$, among all groups, 3.0-mm Omnichroma specimens had significantly higher CAP-I values, while 2.0-mm Filtek Universal Restorative specimens had the lowest values $(P<0.05)$. After 1 month, all restorative materials had CAP-I values greater than 0.20 at both thicknesses. The 2.0-mm and 3.0-mm Estelite Sigma Quick specimens had statistically higher CAP-I values, whereas 2.0-mm Filtek Universal Restorative specimens had the lowest values $(P<0.05)$. CAP-I values at 1 month were significantly higher than those at $24 \mathrm{~h}$ in all tested restorative material groups.

When the effect of cavity depth on CAP-I was evaluated, values were significantly higher for the $3.0-\mathrm{mm}$ groups than for the $2.0-\mathrm{mm}$ groups $(P$ $<0.05$ ) for all tested materials and both durations (Table 3 ).

\section{Discussion}

In esthetic dentistry, the term blending effect (BE) is used to describe the color interaction between dental hard tissues and restorative materials. When they are evaluated together, it expresses the small perceptual and physical color differences between them $[8,13]$. This term is important for clinicians, as it helps in minimizing color mismatches in restorations and ensuring satisfactory shades for dental materials. The BE of dental materials is multifactorial. A previous study found that CAP is not only a perceptional phenomenon [2]; it is also affected by the optic characteristics of surrounding dental tissues and by the color and translucency of dental materials $[14,15]$. To obtain quantitative results only, the present in vitro study therefore investigated the instrumental color adjustment potential of the tested resin composites. However, it is important to remember that $\mathrm{CAP}$ is the interaction between the physical and perceptual components of a blending.

Previous studies have reported that CAP was shade dependent $[8,13]$. In this study, the tested shade of the denture teeth and the color of Estelite Sigma Quick and Filtek Universal Restorative had a Vita shade of A3. To standardize the base color, denture teeth were chosen in this study. However, even if natural teeth are extracted, they show a multilayered, polychromatic structure, which could affect the BE and results obtained. For this reason, the present results may be attributable to the color of both the denture teeth and resin composite used. The color of a resin composite depends on its surrounding tissues, which might explain the better longterm CAP-I results of denture teeth fabricated from Estelite Sigma Quick and Filtek Universal Restorative.

Structural color mechanism is a term used to describe how materials exhibit different colors as a result of their complex structure, not because of their pigmentation [16]. Under such conditions, color comes from the photonic crystals. These crystals are produced by optical control and manipulation because of the response of the materials themselves, which is augmented or diminished by application of light at different wavelengths. In nature, this pattern is observed in the magnificent colors of butterflies, many beetles, and peacocks, all of which are a consequence of natural photonic crystals [17].

The manufacturer of the restorative material Omnichroma indicates that it does not contain pigments but exhibits wide-range color-matching ability, including all Vita classical shades A1-D4, with a single shade. It provides these color characteristics by using smart chromatic technology obtained with a suprananospherical filler of silicon dioxide and zirconium dioxide of uniform 260-nm particles. The monomer has the exact size and shape to generate a red-to-yellow color after polymerization, without the need for additional pigments. This is consistent with previous studies that reported a relationship between translucency reflecting the surrounding dental tissues and the BE $[13,18]$. In this study, Omnichroma had acceptable CAP-I values at $24 \mathrm{~h}$ and 1 month for both thicknesses, while Estelite Sigma Quick, from the same manufacturer, had lower values. Although Estelite Sigma Quick has more filler content than Omnichroma, the particle size and uniformly dispersed particles of Omnichroma may affect the color properties of the material. These results may also be related to the color and nature of the selected denture tooth and to the physical and chemical characteristics of the restorative material, such as monomer structures, fill- 
ers, and oxidation of the carbon-carbon double bonds. In a previous study, Omnichroma had the most significant CAP values [3], perhaps attributed to the restorative materials used in that study and shades investigated.

The effect of filler content, shape, size, and resin matrix on the color parameters of resin composites has been evaluated. Translucency was directly related to the filler content of the materials $[19,20]$. In the present study, the filler content of resin composites ranged from 82 to $76.5 \mathrm{wt} \%$, according to the manufacturer. Suh et al. concluded in their study that a filler content of $80 \%$ or higher significantly affected the BE [20]. Another study reported a strong correlation between translucency and blending effect [15]. In addition, translucency was greater for composites with, than for those without, bisphenol A diglycidyl methacrylate (Bis-GMA) [19]. Estelite Sigma Quick contains Bis-GMA monomer in its resin matrix and a filler content of more than $80 \mathrm{wt} \%$, which could explain its higher translucency and CAP-I, as compared with other pigmented restorative materials. At the same restoration depth, CAP-I was lower for Filtek Universal Restorative than for Omnichroma and Estelite Sigma Quick at both intervals in this study but was acceptable at 1 month.

Although no study has evaluated the effect of restoration depth on CAP, a decrease in cavity size may positively affect the $\mathrm{BE}$ because of changes in the optical properties of the remaining tooth structures $[8,13]$. An increase in thickness of the restoration, regardless of restoration size, enables the restorative materials to better reflect its color properties. When the effect of restoration depth on CAP-I was compared in the same restorative materials in the present study, CAP-I values were higher for $3.0-\mathrm{mm}$ restorations than for 2.0-mm restorations; thus, this part of the null hypothesis was accepted.

No study has investigated the association of time after restoration with CAP. This study assessed 2 durations after restoration. After 1 month, all restorative materials had acceptable CAP-I values at both depths, which was not true at $24 \mathrm{~h}$. Therefore, this part of the null hypothesis was also accepted. These findings explain why the color properties of a material may change over time after a restoration is performed and why satisfactory esthetic outcomes and color stability of the restorations can be achieved with time [21]. Under clinical conditions, multiple factors influence the color matching of restorative materials, including restoration area, restoration depth, amount of surrounding dental tissues, and material-dependent factors such as type of photoinitiators and activators, monomers, filler types, and size [21].

The present universal resin composites exhibited different CAP-I values at $24 \mathrm{~h}$ after restoration, and greater filler content was associated with better results. These findings indicate that, after storage in distilled water for 1 month, CAP-I was satisfactory for all the tested groups and the increase in CAP-I was associated with restoration depth in all restorative materials. The color adjustment potential of a restorative material is an important esthetic feature. When making this evaluation, clinical conditions, patients' expectations, and the operators' professional skills should be kept in mind. Future studies should evaluate the color parameters of these universal materials in natural teeth, both in vivo and in vitro, with a wider range of shades and time intervals.

\section{Conflict of interest}

The authors declare no conflict of interest.

\section{References}

1. Ardu S, Duc O, Di Bella E, Krejci I (2017) Color stability of recent composite resins. Odontology 105, 29-35.

2. Trifkovic B, Powers JM, Paravina RD (2018) Color adjustment potential of resin composites. Clin Oral Investig 22, 1601-1607.

3. Pereira Sanchez N, Powers JM, Paravina RD (2019) Instrumental and visual evaluation of the color adjustment potential of resin composites. J Esthet Restor Dent 31, 465-470.

4. Kim D, Park SH (2018) Color and translucency of resin-based composites: comparison of a-shade specimens within various product lines. Oper Dent 43, 642-655.

5. Radz GM (2013) Composite resins in 2013: an update on their progress. Compend Contin Educ Dent 34, 48-51.

6. Dietschi D, Fahl N Jr (2016) Shading concepts and layering techniques to master direct anterior composite restorations: an update. Br Dent J 221, 765-771.

7. Shinoda H, Ikeda M (2004) Color assimilation on grating affected by its apparent stripe width. Color Res Appl 29, 187-195.

8. Paravina RD, Westland S, Imai FH, Kimura M, Powers JM (2006) Evaluation of blending effect of composites related to restoration size. Dent Mater 22, 299-307.

9. Choi JH, Park JM, Ahn SG, Song KY, Lee MH, Jung JY et al. (2010) Comparative study of visual and instrumental analyses of shade selection. J Wuhan Univ Technol Mater Sci 25, $62-67$

10. Sampaio CS, Atria PJ, Hirata R, Jorquera G (2019) Variability of color matching with different digital photography techniques and a gray reference card. J Prosthet Dent 121, 333-339.

11. Lee YK, Powers JM (2005) Comparison of CIE lab, CIEDE 2000, and DIN 99 color differences between various shades of resin composites. Int J Prosthodont 18, 150-155.

12. de Abreu JLB, Sampaio CS, Benalcázar Jalkh EB, Hirata R (2021) Analysis of the color matching of universal resin composites in anterior restorations. J Esthet Restor Dent 33, 269-276.

13. Paravina RD, Westland S, Kimura M, Powers JM, Imai FH (2006) Color interaction of dental materials: blending effect of layered composites. Dent Mater 22, 903-908.

14. Paravina RD, Westland S, Johnston WM, Powers JM (2008) Color adjustment potential of resin composites. J Dent Res 87, 499-503.

15. Iyer RS, Babani VR, Yaman P, Dennison J (2021) Color match using instrumental and visual methods for single, group, and multi-shade composite resins. J Esthet Restor Dent 33, 394-400.

16. Hiroyuki S, Mitsuo (2004) Color assimilation on grating affected by its apparent stripe width. Col Res App 29, 187-195.

17. Ding Y, Xu S, Wang ZL (2009) Structural colors from Morpho peleides butterfly wing scales. J Appl Phys 106, 074702.

18. Abdelraouf RM, Habib NA (2016) Color-matching and blending-effect of universal shade bulk-fill-resin-composite in resin-composite-models and natural teeth. Biomed Res Int, 4183432.

19. Azzopardi N, Moharamzadeh K, Wood DJ, Martin N, van Noort R (2009) Effect of resin matrix composition on the translucency of experimental dental composite resins. Dent Mater 25, 1564-1568.

20. Suh YR, Ahn JS, Ju SW, Kim KM (2017) Influences of filler content and size on the color adjustment potential of nonlayered resin composites. Dent Mater J 36, 35-40.

21. Çelik EU, Aladağ A, Türkün LŞ, Yılmaz G (2011) Color changes of dental resin composites before and after polymerization and storage in water. J Esthet Restor Dent 23, 179-188. 